\title{
Applications of laser ultrasonic technique on nondestructive testing and evaluation of materials
}

\author{
Yang Zhao ${ }^{1,2^{*}}$, Jianwei Chen ${ }^{1}$, JihuaSun $^{1,2}$, Jiangfeng Song $^{1}$, Jian Ma $^{1}$, Shuai Liu, ${ }^{1}$ and Yinian Zhu ${ }^{2}$ \\ ${ }^{1}$ Laser institute, Qilu University of Technology (Shandong Academy of Sciences), China \\ ${ }^{2}$ Center for Quality Engineering and Failure Prevention, Northwestern University, Evanston, IL 60208, USA
}

\begin{abstract}
Nondestructive testing (NDT) is a wide group of analysis techniques used in science and industry to evaluate the properties of a material, component or system without causing damage. As one of unconventional NDT technology, laser ultrasonic technique (LUT) have played an important role in inspecting the materials in some extreme environments, such as high temperature, high pressure, corrosion, radioactive and etc. LUT uses lasers to generate and detect ultrasonic waves in a noncontact way. Thus, compared with the traditional ultrasonic technique, LUT has the advantages of high precision, high sensitivity without requirement for geometry of target material. So far, LUT has been well applied to composite inspections for the aerospace industry and on-line hot tube thickness measurements for the metallurgical industry. This talk describes the application of LUT on flaws detection in rail and thermal barrier coatings.
\end{abstract}

\section{INTRODUCTION}

Nondestructive testing technique (NDT) is an indispensable basic technology in product quality control and safety. Ultrasonic technology as an indispensable tool for nondestructive testing (NDT) and structure health monitoring (SHM) of materials has been widely used in the fields of aerospace, steel, railway and nuclear power, due to the advantages such as large penetration ability, high detection sensitivity, and easy to realize automatic detection. Laser-based ultrasonic (LBU) allows the same information to be collected by lasers. Generation is accomplished by illuminating the target material with a short-pulse laser [1]. Typically, nanosecond laser pulses are used to generate ultrasonic sources in the megahertzrange for the thickness measurement and defect inspection of the bulk materials, while femtosecond laser systems are necessary for $\mathrm{GHz}$ range which is suitable to evaluate the thin layer in the order of micrometer even nanometer [2]. The detection of ultrasound generated by pulsed laser can be carried out by means of noncontact methods via optical interferometer, optical beam deflection (GCLAD) or knife edge detection [3]. Optical detection of ultrasound is attractive because it can provide absolute measurement of ultrasonic signal with high detection bandwidth in a noncontact way.

In this paper, some experimental study on LBU and hybrid LBU was introduced for various applications. Especially, a novel FBG sensing system developed by us is firstly to use as discrete sensing elements to detect the ultrasonic generated by pulsed laser. At last, we summarized the advantage and drawbacks of above techniques, and discussed the development direction of the LBU technology in the future.

\section{RAIL INSPECTION BY USING LASER-EMAT TECHNIQUE}

A laser-EMAT system used to inspect surface flaw includes a pulsed laser, optical fiber and its coupler, EMAT sensor of shear wave, signal acquisition and processing system (As seen in Figure 1).For the generation of Rayleigh wave, a Q-switch pulsed Nd:YAG laser of wavelength $1064 \mathrm{~nm}$ was used, operating with $10 \mathrm{~ns}$ pulse width. Optical fiber system was employed to transmit the laser. Coupling efficiency of the system approximates $60 \%$, and the maximum pulse energy deposited on the rail surface is about $48 \mathrm{~mJ}$ per pulse.P60 rails with rough surface were selected to prepare the samples of artificial surface defects. The distance $d_{t}$ between the laser spot and surface crack is 55 $\mathrm{mm}$. In the present work, surface defects were detected by observing their interaction with Rayleigh wave. We used EMAT sensor to defect shear wave component from the mode conversion when the Rayleigh wave propagates through the surface crack. It is easy to determine the approximate location of surface defect through monitoring the shear wave. Further, the amplitude of the shear wave will achieve to the maximum value when the flaw is positioned under the EMAT sensor. Then, the location of surface defect can be accurately determined [4]. 


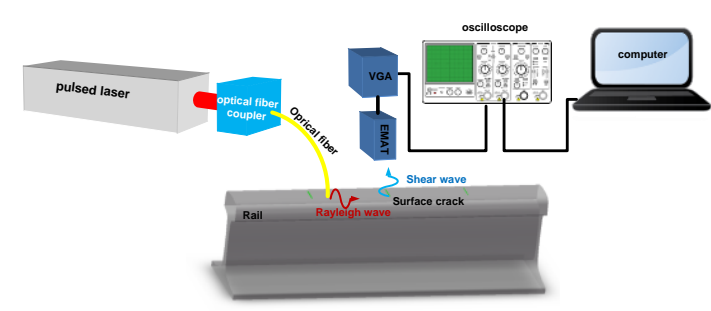

Figure 1: Schematic diagram of Laser-EMAT system used to detect flaw in Rail

Figure 2 illustrates an original A-scan waveform received by the EMAT sensor, which shows time interval approximates $18.15 \mathrm{~s}$ for receiving the shear wave. Then, the distance of $\mathrm{d}=54.45 \mathrm{~mm}$ was obtained based on Eq. (1), which leads to the relative error of inspection is about $1 \%$ calculated by Eq. (2).

$$
\begin{array}{r}
d=c_{R} \times \Delta t \\
\text { error }=\frac{d-d_{t}}{d_{t}} \%
\end{array}
$$

where $d$ is the distance between laser spot and EMAT receiver, $c_{R}$ is the velocity of Rayleigh wave, and $\Delta t$ is the flight time of Rayleigh wave. In this work, $c_{R}$ is about $3000 \mathrm{~m} / \mathrm{s}$.

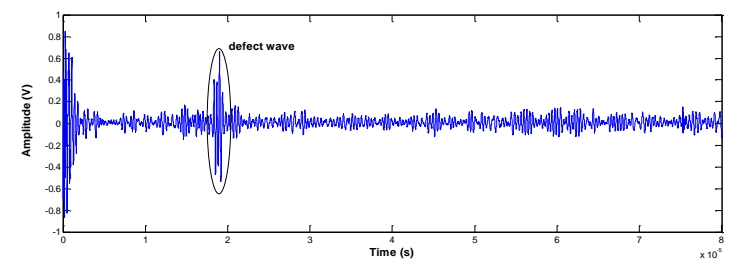

Figure 2: Shear wave from the surface crack due to wavemode conversion

\section{FLAW DETECTION BY USING LASER-FBG SENSING SYSTEM}

An all-fiber adaptive FBG sensing demodulation system that is developed to detect the ultrasonic signal (as seen in Figure 3). The central wavelengths of the tunable DFB semiconductor laser and FBG are $1545.35 \mathrm{~nm}$ and $1550.36 \mathrm{~nm}$, respectively. Optical output power of the semiconductor lasers is around $150 \mathrm{~mW}(\sim 13.19 \mathrm{dBm})$ at $25^{\circ} \mathrm{C}$. The PID controller is continuously employed to control the wavelength of tunable DFB semiconductor laser and to keep it to be locked to the middle-reflection wavelength of the FBG. Then, the same pulsed laser and FBG sensing system was combined to inspect the artificial surface crack with dimension of $50 \mathrm{~mm} \times 1 \mathrm{~mm}$ $\times 1 \mathrm{~mm}$ on the aluminum plate (as seen in figure 9). The water film was covered at the location of laser beam on the surface of aluminum plate so that the energy of Rayleigh wave generated by laser can be increased as much as possible.

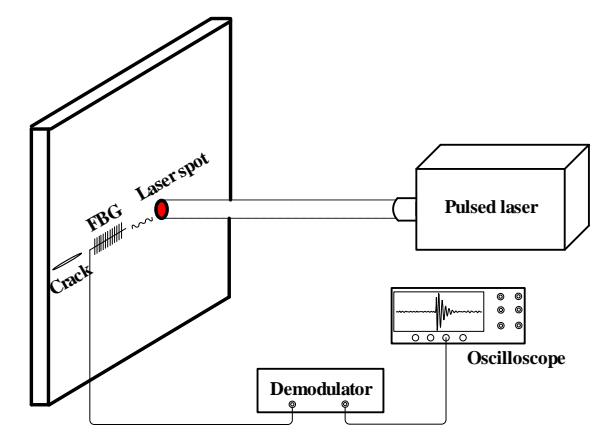

Figure 3: Schematic diagram of hybrid laser ultrasonic testing the surface defect

Next, an aluminum plate that contains an artificial surface crack was detected by the proposed method above. The distance between the crack tip and FBG sensor is about $25 \mathrm{~mm}$. The crack was located by time flight method of the diffraction wave from the Rayleigh wave to meet the crack. Both of the Rayleigh signals with surface crack and without any defect detected by FBG sensor are shown in Figure 4. Thus, the time that receives the diffracted wave by FBG sensor is about 18.5 s. When the velocity of Rayleigh wave in aluminum is 2940 $\mathrm{m} / \mathrm{s}$, the distance between the crack tip and FBG sensor is $27 \mathrm{~mm}$ which is calculated by Equation (1). The relative error between the ultrasonic testing value and the real one approximates $8 \%$.

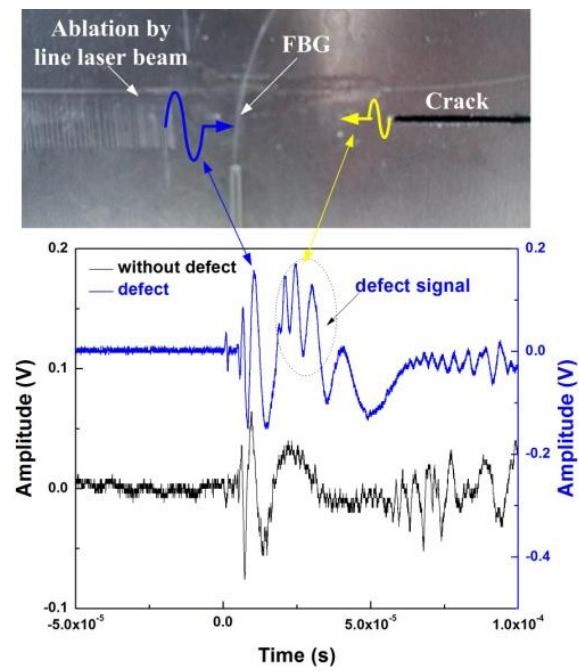

Figure 4: Ultrasonic testing signals (bottom) from the artificial surface crack in the aluminum plate (top)

\section{NDT OF TGO BY LASER ULTRASONIC}

A schematic of the experimental setup used for TF measurements of TBC is shown in Figure. 5. A Qswitched Nd:YAG laser with wavelength of $1064 \mathrm{~nm}$ and pulse duration of $10 \mathrm{~ns}$ was used to generate longitudinal wave in TBC, and the ultrasonic signals were detected by the AIR-1550-TWM interferometer. The laser beam was focused on the TBC specimen using the lens with focal length of $50 \mathrm{~mm}$. Experiments were carried out with applying energy up to $50 \mathrm{~mJ}$ per pulse. To ensure the repeatability of the ultrasonic wave received by the 
interferometer, the location of laser probe was automatic adjustment accurately through combining the step controller with computer. The energy of laser was adjustable so that ultrasonic generation not only occurred in the thermoelastic regime but also had the energy large enough. A TDS5400 digital oscilloscope was employed to record the output signals from the photodetector after averaging over 32 acquisitions in order to reduce random noise.

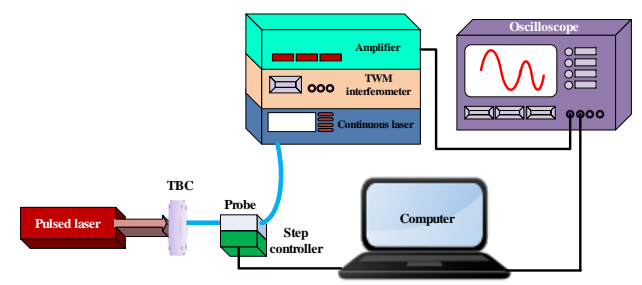

Figure 5: Experimental setup for laser generation and detection of ultrasonic wave

Typical time domain signals of TBC before oxidation and GH3030 substrate are shown in Figure. 6. It can be clearly observed echoes from the front and back surfaces of the coating overlapped for TBC compared with that of substrate.

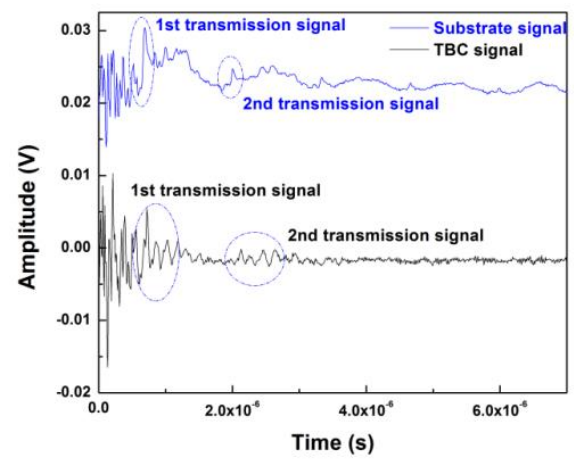

Figure 6: Ultrasonic transmission signals of TBC and substrate

Then, wavelet denoising method was applied to the signal of TBC before and after oxidation respectively (As seen in Figure 7).
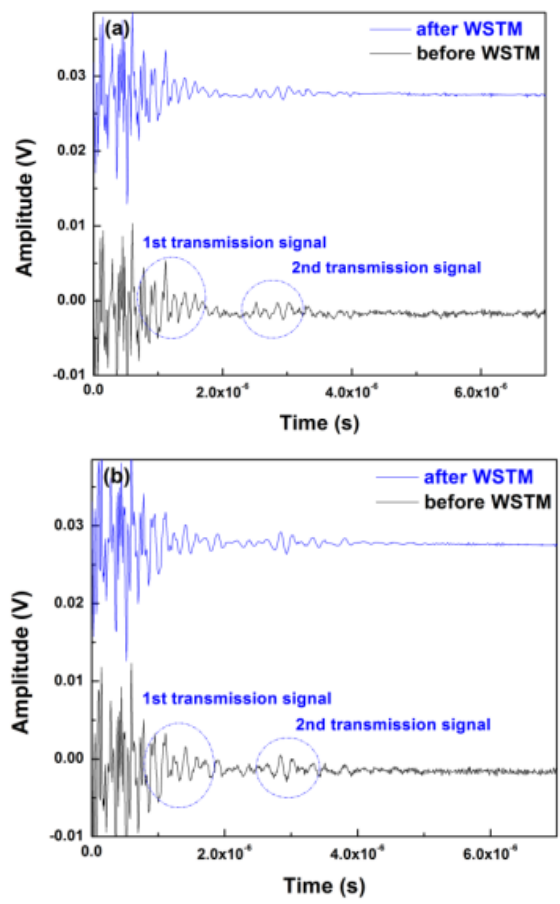

Figure 7: Comparison of Ultrasonic signals after WSTM: (a) before oxidation; (b) after oxidation.

Next, experimental transfer functions of TBC before and after oxidation are obtained, and meanwhile the theoretical ones were given based on the method in Reference [5] (As seen in Figure 8). The experimental transfer functions of TBC before oxidation distinguishes from that of TBC after oxidation. Especially, the amplitude of $H(f)$ in the high frequency range decrease for the TBC after oxidation, which imply that the attenuation corresponding to the frequency increase as the operating time of oxidation. Moreover, the experimental results are basically in accord with the theoretical ones, which show the TGO exist in the TBC after oxidation. To prove this assumption, the section images of the specimens inspected by laser ultrasonic above were obtained by the SEM. Then, TGO with thickness of about $4 \mu \mathrm{m}$ was found in Figure 9. 

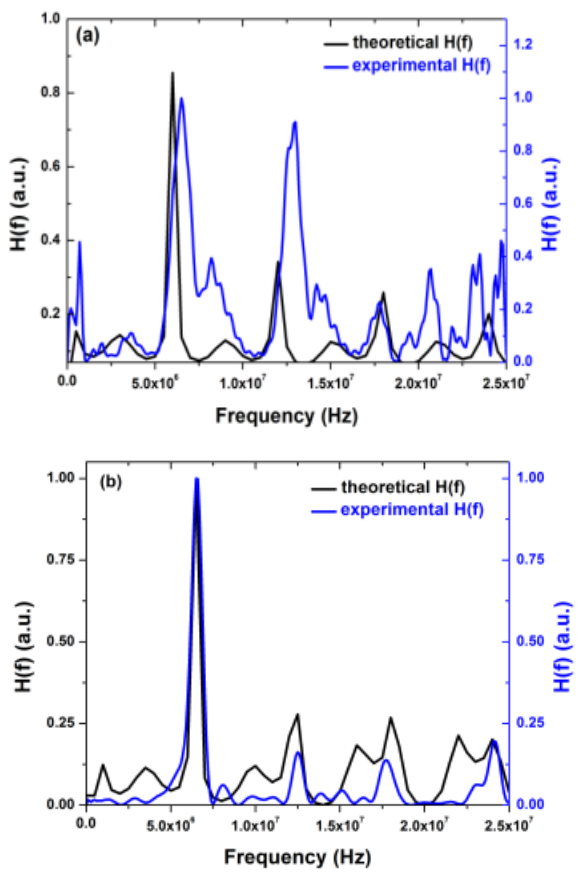

Figure 8: Experimental and theoretical |T(f)|of TBC: (a) before oxidation; (b) after oxidation

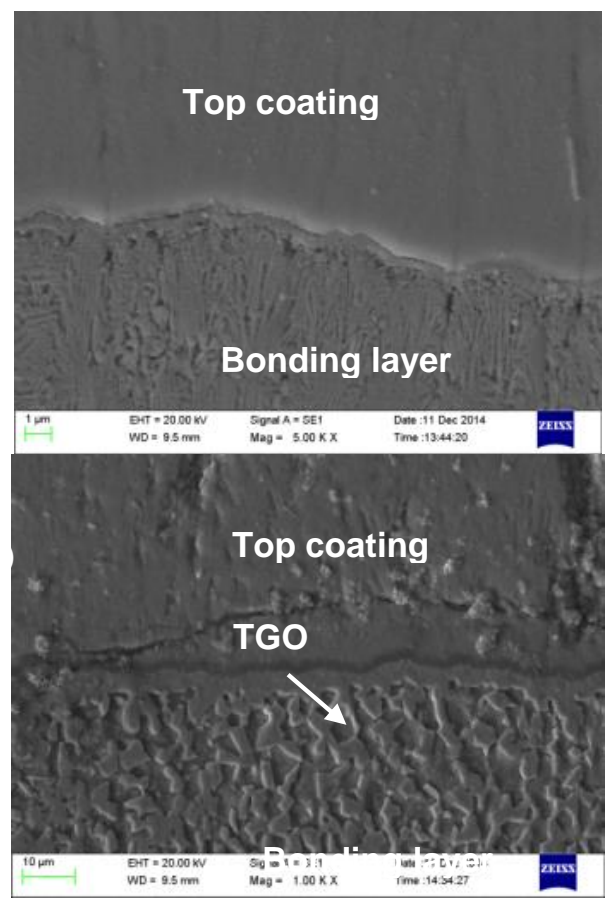

Figure 9: Cross-sectional microstructure of the TBC: (a) before oxidation; (b) after oxidation

\section{CONCLUSION}

LBU technology is the main development direction in the field of NDT research and applications. LBU is also a useful method for detection in extreme environment or to special piece. Various LBU technologies in recent years that has the application prospect is reviewed, and in particular emphasizes on hybrid LBU technology. LaserEMAT detecting technology works without coupling agent, and is not limited by the shape and surface roughness of the tested material. This technology has the broad application prospect and huge application value in on the NDT of metal materials at high speed operation and structures. This hybrid technique saves a lot of cost compared with LBU above, which is feasible for inspection of rail. Compared with the two LBU techniques above, laser-FBG sensing technique is novel for the NDT of materials and SHM of structures. This hybrid LBU is considered to be most promising for NDT and SHM applications, especially in infrastructure damage detection.Future research will be focused on the development of multi-channel all-fiber adaptive FBG sensing system that is more compact and cost effective for the purpose of practical application.

\section{ACKNOWLEDGE}

The financial support for this research work was provided in part by the Natural Science Foundation of Shandong Provence under Award No. ZR2017QEE002, the Primary Research \& Development Plan of Shandong Province under Award No. 2016ZDJQ0401 and the Key technology for prevention and control of serious accidents in production safety under Award No.Shandong-0001-2017AQ.

\section{REFERENCES}

1. Zhao, Y., Chen, J., Zhang, Z., 2015. Nondestructive Characterization of Thermal Barrier Coating by Noncontact Laser Ultrasonic Technique. Opt. Eng., 54(9):094104.

2. .Krishnaswami, S., 2003. Theory and Applications of Laser-ultrasonic Techniques. Ultrasonic Nondestructive Evaluation: Engineering and Biological Material Characterization, Inc., pp.435494.

3. Zhao, Y., Sun, J. H., Ma, J., et al, 2014. Application of the Hybrid Laser Ultrasonic Method in Rail Inspection. Insight-NDT. CM., 56(7):360-366.

4. Zhao, Y., Cui, L., Sun, J., et al, 2014. Hybrid Laser Ultrasonic Inspection Based on Optical Fiber Technique. Chin. Opt. Lett., 12(s1):11102.

5. Zhao, Y., Chen, J. W., Zhang, Z. Z., Zhu, Y. N. (2015, October). Nondestructive evaluation of residual stress and TGO by using laser ultrasonic method. In Piezoelectricity, Acoustic Waves, and Device Applications (SPAWDA), 2015 Symposium on (pp. 411-417). IEEE. 